\title{
Attitude of nurses and midwives towards collaborative care with physicians in Jimma University medical center, Jimma, South West Ethiopia
}

\author{
Eneyew Melkamu* (i) and Aynalem Yetwale
}

\begin{abstract}
Background: Good attitude on collaborative care between nurses and midwives with physicians is crucial for better team working. This further enables those vital health care professionals to provide quality and improved care for their clients.

Objective: To assess the attitude of nurses and midwives towards collaborative care with physicians in Jimma University medical center, Jimma, South West Ethiopia.

Methodology: The institution-based cross-sectional study was conducted from March 20 to April 8, 2019, using a semi-structured and standardized questionnaire. Study units were selected by simple random sampling using the lottery method. A total of 410 participants were included in the study. Data were entered into Epi data version 4.2 and exported to statistical packages for social sciences version 23 for cleaning and further analysis. Descriptive statistics were presented with tables, figures, and narratives. The level of significance was set at a $p$ value of less than 0.05 in multivariable logistic regression.
\end{abstract}

Results and conclusion: More than five out of ten, 234 (57.2\%) of participants had a good (good attitude is defined in the operational definition section of methodology) attitude towards collaborative care with physicians, and the rest 175 (42.8\%) poor attitude toward it. Participants had the highest median score in the shared education and teamwork (26.0) subscale and midwives were found to have higher mean rank scores compared to nurses. It was only in the nurse's/midwife's autonomy subscale that a statistically significant difference was found $(Z=-2.92, p$ value $=0.003)$. More generally, more than have of the participants had a good attitude on providing care collaboratively with physicians, though a significant proportion of nurses and midwives also rate collaborative care provision with physicians as poor.

Recommendations: The findings of this study suggested that interventions are needed to be taken to improve nurse's and midwife's attitudes on the provision of collaborative care with physicians. Enhancement of shared education, cooperation rather than dominance and caring attitude are all vital. These all could in turn enhance the quality of care provided for clients.

Keywords: Attitude, Nurses, Midwives, Physicians, Collaborative care

*Correspondence: enemelkamu@gmail.com

School of Midwifery, Faculty of Health Sciences, Institute of Health, Jimma University, Jimma, Ethiopia

\section{Background}

According to Carnwell and Buchanan (2004), collaborative care is defined as "an intellectual and co-operative endeavor, knowledge and expertise more important original author(s) and the source, provide a link to the Creative Commons licence, and indicate if changes were made. The images or other third party material in this article are included in the article's Creative Commons licence, unless indicated otherwise in a credit line to the material. If material is not included in the article's Creative Commons licence and your intended use is not permitted by statutory regulation or exceeds the permitted use, you will need to obtain permission directly from the copyright holder. To view a copy of this licence, visit http://creativecommons.org/licenses/by/4.0/. The Creative Commons Public Domain Dedication waiver (http://creativeco mmons.org/publicdomain/zero/1.0/) applies to the data made available in this article, unless otherwise stated in a credit line to the data. 
than role or title, joint venture, team working, participation in planning and decision making, a non-hierarchical relationship, sharing of expertise, willingness to work together towards an agreed purpose, trust and respect in collaborators, highly connected network, and low expectation of reciprocation" [1].

Health care provision needs many interactions and collaborations between different healthcare professionals with varying levels of education and professional qualifications [2]. Collaborative care benefits both care providers and consumers. Benefits for the care providers include: increased professional satisfaction due to clearer, more consistent goals of care and improved communication with other providers, enables the provider to learn new skills and approaches to care and work to full scope, provides an environment for innovation, and allows providers to focus on individual areas of expertise [3]. Benefits for the patient include: improves care by increasing the coordination of services, integrates health care for a wide range of health needs, empowers consumers as active partners in care, and results in better patient outcomes [3].

Though it was not possible to find studies assessing attitudes of nurses and midwives towards collaborative care with physicians at once (the same study assessing nurse's and midwife's attitude at the same time), studies conducted separately in different settings have shown that both nurses and midwives are short of good attitude [4-7]. A study conducted in Nepal indicated that only quarter of nurses $(25.3 \%)$ had a good attitude towards collaborative care with physicians [4]. On the other side, in a study conducted in Saudi Arabia nurses were found to have better scores on the Jefferson scale of attitudes towards nurse-physician collaboration (JSATNPC) with a mean score of $51.2 \pm$ SD 5.46 from the possible maximum score of 60 showing that nurses had a positive attitude towards working collaboratively with physicians[8].

Other studies undertaken in Germany and Malaysia had shown that nurses fail to have a positive or good attitude on their collaboration with physicians (more than half of nurses in both studies) said that they had a poor attitude on this aspect $[9,10]$. The other study conducted in the North-Western part of Ethiopia also indicated that less than half (41\%) of nurses had a poor attitude towards collaborating with physicians and had higher mean score on the shared education and teamwork subscale, $23.33 \pm$ SD 3.07, and lowest score in the physician's dominance subscale, $5.87 \pm$ SD 1.81[11].

Concerning midwives, it is difficult to find works of literature or documents which had assessed midwife's attitude towards collaborative care with physicians especially using the JSATNPC measuring scale. But, looking at a few works of the literature undertaken in different settings, midwives also face collaborative problems with physicians and found to have negative attitudes towards collaborative care with physicians. a systematic review which had assessed studies conducted in the United States (US), United Kingdom (UK) and Australia had shown that poor (negative) attitude towards collaborative care with physicians was a problem [12].

One more study done in the Netherlands showed that nurses and midwives rated their view towards collaboration with a physician as poor indicating that they both lack one core element for multidisciplinary quality health care, having a good attitude [13].

On top of the effort the government is taking to raise the number and quality of health services, it is vital to have a good attitude of nurses and midwives with the physician to provide collaborative care and thus enhancing the quality of care and making the work environment smooth and friendly. But, to the best of the author's knowledge, regarding the attitude of nurses and midwives on collaborative care with physicians, particularly those of midwives are not well explored and inadequate to notify health care providers and other concerned bodies in Ethiopia. Therefore, this study tried to fill these holes and examined the problem better.

\section{Methods \\ Aims of the study}

1 To assess the attitude of nurses and midwives on collaborative care with physicians at Jimma University medical center, Jimma, South West Ethiopia.

2 To explore the possible difference between nurses and midwives in attitude towards a collaborative care with physicians, at Jimma University medical center, Jimma, South West Ethiopia.

\section{Study area, period and population}

This institution-based cross-sectional study was conducted from March 29 to April 12, 2019 G.C in Jimma University medical center which is located in Jimma town $352 \mathrm{~km}$ southwest of the capital of Ethiopia, Addis Ababa. Currently, it is the largest and the only teaching and referral hospital in the southwestern part of the country, providing services for approximately 15 million people in its catchment area.

It has 1600 staff of whom 648 are nurses and midwives (566 nurses and 82 midwives). The hospital also has 800 beds and provides many health care services in the gynecology and obstetrics, internal medicine, pediatrics, emergency, radiology, surgery, and other departments.

The study population was nurses and midwives with a length of service half a year and above (as they are 
considered to have more experience of working with physicians in addition to being considered full employees by the civil service law of Ethiopia) and those available during the study period.

\section{Sample size and sampling procedure}

The sample size $(n)$ was calculated using the formula to estimate a single population proportion:

$n=[(\mathrm{Z} \alpha / 2) 2 \mathrm{p}(1-\mathrm{p}) / \mathrm{d} 2]$. Then the minimum sample size: $n=(1.96) 2(0.41)(0.59) /(0.05) 2=371.71 \approx 372$ taking $p=0.41$ from a previous study done in North West Ethiopia [11].

Adding 10\% for non-response rate, the final sample size was calculated to be: $n=372+37.2 \approx 410$. Using population proportion formula: $\mathrm{ni}=\mathrm{Ni} \times \mathrm{n} / \mathrm{N}$, number of nurs es $=566 \times 410 / 648=358.12 \approx 358$ and number of midwives $=82 \times 410 / 648=51.88 \approx 52$. Therefore, a total of 358 nurses and 52 midwives were included in the study.

A stratified sampling technique was used to select the study population. The study population was stratified by profession to nurses and midwives and the sample was taken from each stratum proportionally. Individual participants were selected using simple random sampling with a lottery method to attain the final sample size. A list of nurses and midwives from each ward was used as a sampling frame.

\section{Operational definitions Good attitude of nurses and midwives towards collaborative care with physicians}

Higher factor score on the adapted Jefferson scale of attitude towards nurse-physician collaboration (55 and above).

\section{Poor attitude of nurses and midwives towards collaborative care with physicians}

Lower factor score on the adapted Jefferson scale of attitude towards nurse-physician collaboration (below 55).

\section{Data collection instrument and procedure}

Data collection was facilitated by five trained data collectors who have a BSc degree. The sociodemographic characteristics were assessed using close-ended questions. The attitudes of nurses and midwives were assessed by the adapted version of the Jefferson Scale of Attitudes toward Nurse Physician Collaboration. The final version of the JSAPNC contains 15 items answered on a 4-point Likert-type scale from ( 1 "strongly disagree" to 4 "strongly agree"). A higher total score reflects a more positive attitude toward physician-nurse collaborative relationships". The instrument has known factors that were identified as: "'shared education and teamwork' (7 items), 'caring as opposed to curing' (3 items), 'nurse's autonomy' (3 items) and 'physician's authority'(2 items).

The tool was originally developed by Hojat and Herman in 1985 and was modified in 2003 by Hojat et al. [14]. This tool was supported by psychometric evidence including construct validity and internal consistency reliability that can be used as a research tool in western countries. According to Hussein SZ1, Fatin Amira Ahmad and S.Hawa M.Noh (2018), the tool has good internal consistency with a Cronbach's alpha coefficient reported of 0.87. In the current study, it was found to have a Cronbach's alpha coefficient of 0.72 which is within the acceptable range. To check whether it works in Ethiopia, a pretest was conducted by a study done in North West Ethiopia in Goba referral hospital and finally confirmed that the tool can be applied in the Ethiopian context [11]. This tool (JSAPNC) was preferred from other psychometrically supported instruments available for measuring nurse's and midwife's attitude on collaborative care with physicians as it was applied in the Ethiopian context and confirmed to be valid and reliable to be used in Ethiopian studies.

Data were collected by administering a written questionnaire to study participants which were prepared originally in English and then translated into Affann Oromo and Amharic (the local languages in the study area) by a language expert in all those three languages. Then, it was translated back to English to keep its consistency and validity.

\section{Data quality control}

To maintain the quality of the data, a pretest was done on $41(10 \%)$ of nurses and midwives in a different hospital found in Jimma city, South West Ethiopia, and necessary modifications including wordings were made on the questionnaire before it was applied on the study population. Furthermore, each questionnaire was checked for completeness before data entry. Data were interred in software called Epi data version 4.2 to point out errors made during data collection automatically then transferred to SPSS version 23. Furthermore, training was given to data collectors and supervisors. The overall data collection process was monitored by supervisors.

\section{Data processing and analysis}

The collected data were entered into epi data version 4.2 and exported to SPSS version 23 for cleaning and further analysis. Again, a $p$ value of less than 0.25 in binary and 0.05 in multivariable logistic regressions was considered as significant at a $95 \%$ confidence level. Mann-Whitney $U$ test was used to evaluate the possible difference between nurses and midwives on attitude towards collaborative care with physicians. Results were presented 
by frequency tables, percentages, measures of central tendency, and dispersion and statements.

\section{Results}

Sociodemographic characteristics of study participants

A total of 409 participant data were considered for analysis as there was one incomplete questionnaire yielding a response rate of $99.76 \%$. The mean age of the respondents was 32.14 ( $\mathrm{SD} \pm 7.12$ ) years. The other sociodemographic characteristics of study participants are presented with Table 1.

The attitude of nurses and midwives towards collaborative care with physicians

As indicated in Fig. 1 below, it seems more midwives had a good attitude (explained in the operational definition section of methodology "what is a good attitude towards collaborative care with physicians"). Though, it may be

Table 1 Sociodemographic characteristics of respondents $(n=409)$ in Jimma University medical center, Jimma, South West Ethiopia

\begin{tabular}{|c|c|c|}
\hline Characteristics & Frequency & Percentage \\
\hline \multicolumn{3}{|l|}{ Sex } \\
\hline Male & 234 & 57.2 \\
\hline Female & 175 & 42.8 \\
\hline \multicolumn{3}{|l|}{ Age } \\
\hline $20-29$ & 166 & 40.5 \\
\hline $30-39$ & 183 & 44.6 \\
\hline $40-62$ & 69 & 14.9 \\
\hline \multicolumn{3}{|l|}{ Marital status } \\
\hline Married & 252 & 61.6 \\
\hline Single & 157 & 38.4 \\
\hline \multicolumn{3}{|l|}{ Occupational status } \\
\hline $\begin{array}{l}\text { Ordinary staff (with no manage- } \\
\text { rial role) }\end{array}$ & 393 & 96.1 \\
\hline Head staff & 16 & 3.9 \\
\hline \multicolumn{3}{|l|}{ Length of service in years } \\
\hline $0.5-10.5$ & 332 & 81.2 \\
\hline $10.6-20.6$ & 56 & 13.7 \\
\hline $20.7-32.0$ & 21 & 5.1 \\
\hline \multicolumn{3}{|l|}{ Educational status } \\
\hline \multicolumn{3}{|l|}{ Diploma } \\
\hline Nurse & 81 & 22.7 \\
\hline Midwife & 6 & 11.5 \\
\hline \multicolumn{3}{|l|}{ BSc degree } \\
\hline Nurse & 273 & 76.5 \\
\hline Midwife & 46 & 88.5 \\
\hline \multicolumn{3}{|l|}{ MSc degree and above } \\
\hline Nurse & 3 & 0,8 \\
\hline Midwife & - & - \\
\hline
\end{tabular}

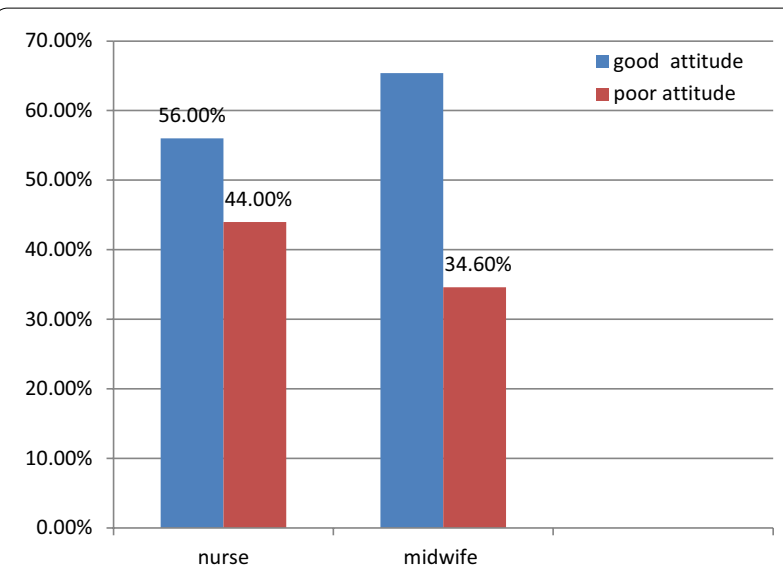

Fig. 1 Classification of the attitude of nurses and midwives towards collaborative care with physicians at Jimma University medical center, Jimma South West Ethiopia. As shown in figure above, around two-thirds (65.4\%) of midwives and more than half (56.0\%) of the nurses had a good attitude towards collaborative care with physicians.

due to the smaller sample size as compared to nurses as the number of nurses is more than six times that of midwives. The rest is shown in Fig. 1 and Table 2.

In Table 3, it is illustrated that none of the participants neither disagree nor strongly disagree to the question "nurses/midwives are qualified to assess and solve psychological aspects of patient needs" and 253 (61.7\%) of them strongly disagree with "doctors should be the primary deciders in all health care matters of the hospital".

As shown in Table 3, nurses and midwives had the highest median score in the "shared education and teamwork" and the lowest score was found in the "physician's dominance/authority" subscale. This showed that nurses and midwives had no positive view of the physician's dominance, but rather prefer to share education and care.

Difference between nurses and midwives on their attitude towards collaborative care with physicians

The difference between nurses and midwives in the attitudes towards collaborative care with physicians was evaluated with the Mann-Whitney U test. This method is used to test for differences between two independent groups on a continuous measure. This test is the nonparametric alternative to the t-test for independent samples. Instead of comparing means of the two groups as in the case of the $t$-test, the Mann-Whitney $U$ test actually compares medians. It converts the scores on the continuous variable to ranks across the two groups. It then evaluates whether the ranks for the two groups differ significantly. As the scores are converted to ranks, the actual distribution of the scores does not matter. 
Table 2 The response of nurses and midwives to the JSANPC (attitude towards collaborative care with physicians) items. Responses of participants on the attitude towards collaborative care with physicians measuring items $(n=409)$ in Jimma University medical center, Jimma, South West Ethiopia

\begin{tabular}{|c|c|c|c|c|}
\hline \multirow[t]{2}{*}{ Items } & \multicolumn{4}{|l|}{ Frequencies } \\
\hline & Strongly agree & Agree & Disagree & Strongly disagree \\
\hline \multicolumn{5}{|l|}{ Shared education and teamwork subscale } \\
\hline $\begin{array}{l}\text { A nurse/midwife should be viewed as a collaborator and colleague with a physician } \\
\text { rather than his/her assistant }\end{array}$ & $320(78.2 \%)$ & $77(18.8 \%)$ & $7(1.7 \%)$ & $5(1.2 \%)$ \\
\hline $\begin{array}{l}\text { Education is needed for physicians to improve collaborative relationships with } \\
\text { nurses/midwives }\end{array}$ & $301(73.6 \%)$ & $92(22.5 \%)$ & $12(2.9 \%)$ & $4(1.0 \%)$ \\
\hline $\begin{array}{l}\text { The educational programs should include areas on team learning and interprofes- } \\
\text { sional collaboration between physicians and nurses/midwives }\end{array}$ & $276(67.5 \%)$ & $127(31.0 \%)$ & $6(1.5 \%)$ & - \\
\hline $\begin{array}{l}\text { Nurses/midwives are responsible for monitoring the progress of treatment the } \\
\text { patient is taking }\end{array}$ & $290(70.9 \%)$ & $107(26.2 \%)$ & $9(2.2 \%)$ & $3(0.7 \%)$ \\
\hline $\begin{array}{l}\text { Nursing/midwifery and medical students should be involved in teamwork during } \\
\text { their education in order to understand their respective roles }\end{array}$ & $265(64.8 \%)$ & $131(32.0 \%)$ & $13(3.2 \%)$ & - \\
\hline Nurses/midwives and physicians share many areas of responsibilities & $310(75.8 \%)$ & $85(20.8 \%)$ & $9(2.2 \%)$ & $5(1.2 \%)$ \\
\hline $\begin{array}{l}\text { Decisions on hospital discharge of patients should be contributed by both physi- } \\
\text { cians and nurses/midwives when the need arises }\end{array}$ & $278(68.0 \%)$ & $123(30.1 \%)$ & $7(1.7 \%)$ & $1(0.2 \%)$ \\
\hline \multicolumn{5}{|l|}{ Physician's authority subscale } \\
\hline Nurses/midwives are primarily responsible to accomplish the physician's orders & $21(5.1 \%)$ & $23(5.6 \%)$ & $125(30.6 \%)$ & $240(58.7 \%)$ \\
\hline Doctors should be the primary deciders in all health care matters of the hospital & $24(5.9 \%)$ & $20(4.9 \%)$ & $113(27.6 \%)$ & $252(61.6 \%)$ \\
\hline \multicolumn{5}{|l|}{ Nurse's/midwife's autonomy subscale } \\
\hline $\begin{array}{l}\text { Nurses/midwives should participate in decisions with the hospital support services } \\
\text { that affects their work }\end{array}$ & $275(67.2 \%)$ & $120(29.3 \%)$ & $10(2.4 \%)$ & $4(1.0 \%)$ \\
\hline Nurses/midwives should be accountable for their care to the patient & $304(74.3 \%)$ & $89(21.8 \%)$ & $11(2.7 \%)$ & $5(1.2 \%)$ \\
\hline $\begin{array}{l}\text { Nurses/midwives should clarify a physician's order when they feel that it might have } \\
\text { the potential for unfavorable effects on the patient }\end{array}$ & $257(62.8 \%)$ & $121(29.6 \%)$ & $25(6.1 \%)$ & $6(1.5 \%)$ \\
\hline \multicolumn{5}{|l|}{ Caring as opposed to curing subscale } \\
\hline $\begin{array}{l}\text { Nurses/midwives should be involved in making policy decisions influencing their } \\
\text { working conditions }\end{array}$ & $279(68.2 \%)$ & $112(27.4 \%)$ & $12(2.9 \%)$ & $6(1.5 \%)$ \\
\hline Nurses/midwives have their own expertise in patient education and counseling & $307(75.0 \%)$ & $98(24.0 \%)$ & $4(1.0 \%)$ & - \\
\hline $\begin{array}{l}\text { Nurses/midwives are qualified to assess and solve psychological aspects of patient } \\
\text { needs }\end{array}$ & $328(80.2 \%)$ & $81(19.8 \%)$ & - & - \\
\hline
\end{tabular}

Table 3 The score of nurses and midwives on each subscale of JSANPC. Scores of participants on each subscale of the JSANPC in Jimma University medical center, Jimma, South West Ethiopia

\begin{tabular}{lllll}
\hline Subscale & Median score & IQR & Minimum score & $\begin{array}{c}\text { Maximum } \\
\text { score }\end{array}$ \\
\hline Shared education and teamwork & 26 & 3 & 16 & 28 \\
Caring as opposed to curing & 12 & 1 & 7 & 12 \\
Nurse's/midwife's autonomy & 11 & 2 & 3 & 12 \\
Physician's dominances/authority & 8 & 2 & 2 & 8 \\
\hline
\end{tabular}

As shown in Table 4, midwives had a higher mean rank in all subscales compared to nurses and it is only in the nurse's/midwife's autonomy that a statistically significant difference was obtained.
Factors associated with the attitude of nurses and midwives towards collaborative care with physicians As indicated in Table 5, nurses and midwives were not significantly different in all variables considered for the 
Table 4 Difference in attitude towards collaborative care with physicians and separate scores of nurses and midwives in Jimma University medical center, Jimma, South West Ethiopia

\begin{tabular}{|c|c|c|c|c|c|c|}
\hline JSANPC subscales & Profession & Median & IQR & Mean rank & Z-value & $p$ value \\
\hline \multirow[t]{2}{*}{ Shared education and teamwork } & Nurse & 26.0 & 3.0 & 201.29 & -1.69 & 0.09 \\
\hline & Midwife & 27.0 & 3.0 & 230.50 & & \\
\hline \multirow[t]{2}{*}{ Caring as opposed to curing } & Nurse & 12.0 & 1.0 & 204.92 & -0.04 & 0.97 \\
\hline & Midwife & 12.0 & 2.0 & 205.54 & & \\
\hline \multirow[t]{2}{*}{ Nurse's/midwife's autonomy } & Nurse & 11.0 & 2.0 & 198.78 & -2.92 & 0.003 \\
\hline & Midwife & 12.0 & 1.0 & 247.69 & & \\
\hline \multirow[t]{2}{*}{ Physician's dominances/authority } & Nurse & 8.0 & 2.0 & 202.56 & -1.19 & 0.23 \\
\hline & Midwife & 8.0 & 2.0 & 221.73 & & \\
\hline
\end{tabular}

Table 5 Binary and multivariable logistic regression of variables associated with the attitude of nurses and midwives towards collaborative care with physicians at Jimma University medical center, Jimma South West Ethiopia

\begin{tabular}{|c|c|c|c|c|c|}
\hline \multirow[t]{2}{*}{ Variable } & \multicolumn{2}{|l|}{ Frequency } & \multirow[t]{2}{*}{ COR, $95 \% \mathrm{Cl}$} & \multirow[t]{2}{*}{ AOR, $95 \% \mathrm{Cl}$} & \multirow[t]{2}{*}{$p$ value } \\
\hline & Good attitude & Poor attitude & & & \\
\hline \multicolumn{6}{|l|}{ Age } \\
\hline $20-29$ & 90 & 76 & 1 & 1 & \\
\hline $30-39$ & 100 & 82 & $1.030,0.675-1.571$ & $1.005,0.635-1.590$ & 0.982 \\
\hline $40-62$ & 44 & 17 & $2.186,1.155-4.135$ & $2.174,0.705-6.703$ & 0.177 \\
\hline \multicolumn{6}{|l|}{ Marital status } \\
\hline Single & 86 & 71 & $0.851,0.569-1.272$ & $1.015,0.651-1.583$ & 0.947 \\
\hline Married & 148 & 104 & 1 & 1 & \\
\hline \multicolumn{6}{|l|}{ Level of education } \\
\hline Diploma & 52 & 35 & $1.143,0.706-1.850$ & $1.241,0.757-2.032$ & 0.392 \\
\hline BSc degree and above & 182 & 140 & 1 & 1 & \\
\hline \multicolumn{6}{|l|}{ Profession } \\
\hline Nurse & 200 & 157 & $0.674,0.367-1.239$ & $0.634,0.340-1.182$ & 0.152 \\
\hline Midwife & 34 & 18 & 1 & 1 & \\
\hline \multicolumn{6}{|c|}{ Occupational status in the hospital } \\
\hline Ordinary staff & 221 & 169 & $0.297,0.083-1.057$ & $0.436,0.115-1.660$ & 0.224 \\
\hline Head staff & 13 & 6 & 1 & 1 & \\
\hline \multicolumn{6}{|l|}{ Length of service in years } \\
\hline $0.5-10.5$ & 181 & 151 & $0.738,0.298-1.827$ & $1.534,0.395-5.954$ & 0.536 \\
\hline 10.6-20.6 & 40 & 16 & $1.538,0.536-4.416$ & $1.809,0.579-5.652$ & 0.308 \\
\hline $20.7-32.0$ & 13 & 8 & 1 & 1 & \\
\hline
\end{tabular}

" 1 " in Table 5 indicates category used as a reference in the analysis

"AOR": adjusted odds ratio

"COR": crude odds ratio

analysis based on the cross-tabulation result.

\section{Discussion}

It is noted that more than half of nurses and midwives had a good attitude towards collaborative care with physicians. But, it could not be forgotten that more than one-third of them also rate their attitude towards collaboration with physicians as poor which is an important figure.

In this study, around five out of ten (56\%) of nurses and nearest to two-thirds (65.4\%) midwives had a good attitude towards collaborative care with physicians. Almost similar findings were obtained with studies conducted in Saudi Arabia, Tehran, and Palestine [7, 15, 16]. A better rating of good attitude was found in a study conducted 
in Nigeria (84\%)[17]. This variation could have resulted from study time variation as today's nurses and midwives are trained in a more harmonized teaching system that may help develop a good attitude to do in collaboration with physicians. Again the difference may come as a result of sample size variations and the point that in this study it is both nurses and midwives attitude towards collaborative care which is evaluated as a single population and this may have influenced the overall attitude of the participants.

Lower figures of attitude towards collaborative care with physicians were noted in studies conducted in Canada, the Netherlands and Ethiopia [11, 18, 19]. These variations may come from the difference in the study area, socio-cultural variations sample size, study period, and the way the data are collected and analyzed as those referenced studies assessed the attitude of nurses and midwives towards collaborative care with physicians separately which could affect the figure obtained. Again, a Nepalese study has also shown that only a quarter of nurses had a good attitude towards collaborative care with physicians $(25.3 \%)$ and on the contrary threefourths of them rate their attitude as poor [4]. These differences may arise from variation in sample size as the Nepal study had a lower sample size than this study. Besides, the inconsistency could be due to a difference in the study area (as in the referenced article participants were from different health facilities), study time and method of analysis used.

In the present study, participants had the highest median score in the shared education and teamwork subscale (26.0) and the lowest median score was recorded in the physician's dominance/authority subscale (8.0). This finding is almost consistent with many of the literatures considered for this study $[6,7,11,16,18,20-22]$. This consistency on the issue in almost all works of literatures is expected. It could be because that human mostly did not need dominance and wants to share things rather than having commanding and subordinate interactions. Thus, the same is true in this case that nurses and midwives prefer to share and work as a team which seems a positive view than being dominated by physicians. Moreover, it is known that it is in the current times that health education curriculums are focusing on multidisciplinary education and teamwork which thus may impact collaborative caretaking in the work environment later on.

Another important finding of this study is that nurses and midwives did not significantly differ in the three subscales of the JSANPC (shared education and teamwork, caring as opposed to curing and physician's dominance/authority) except the nurse's/midwife's autonomy $(p=0.003)$. Nurse's/midwife's autonomy was also a factor (made a significant difference) in studies conducted in
Saudi government hospitals [16]. On the contrary, nurse's autonomy was not a significant factor in the studies done in Mansoura University Hospital, Egypt $(\mathrm{p}=1.854)$ and NorthWest Ethiopia $(p=0.223)[11,20]$. This variation could have resulted from sample size variation, the difference in the study area and analytical method used as it was the independent samples T-test which was used in both the referenced studies. Again, the change in the direction and emphasis of professional autonomy and the presence of clear professional roles and independence could make nurses and midwives value more for their professional autonomy and independence.

\section{Strengths and limitation}

It was based on primary data which could improve the validity of the results obtained. The other strength is that the questionnaire was standardized (adapted standardized questionnaire) which increases the reliability and validity of the instrument. The limitation of the study may be the study setting as the study was conducted in one area that may to some extent border its generalizability.

\section{Conclusion}

It is noted in this study that a higher proportion of nurses and midwives had a good attitude towards collaborative care with physicians. But, it should not be undermined that a significant figure of nurses and midwives also rate their collaborative care attitude as poor. This is a signal that interventions are needed to enhance their attitude as it would finally affect the quality of care provided for the client. A different attitude improving training and the creation of a smooth working environment is recommended. Again, from the four subscales, it was only the nurse's/midwife's autonomy that appeared to significantly differ which is indicative that how much nurses and midwives value their professional autonomy (midwives seem to value their autonomy more as shown with larger mean rank score). There was no variable found to significantly affect a participant's attitude towards collaborative care with physicians. The authors also recognize the need to have a good attitude of physicians towards collaborative working with these two health care providers to create a smooth working environment.

\footnotetext{
Abbreviations

AOR: Adjusted odds ratio; BSc: Bachelor Science; COR: Crude odds ratio; IQR: Inter-quartile range; JSANPC: Jefferson Scale of Attitudes towards Nurse-Physician Collaboration; SPSS: Statistical Packages for Social Sciences.

\section{Acknowledgements}

We would like to remarkably thank Mekelle University for the financial support provided. We are also appreciative of Jimma University medical center staff in general and study participants and data collectors in particular for their cooperation. Last but not least, gratitude goes to colleagues and friends for their inspiration and support throughout the work of this paper.
} 


\section{Authors' contributions}

EM: designed and conceptualized the study, analyzed and interpreted the data, discussed the findings of the study, prepared the manuscript and revised the manuscript. AY: participated in analyzing and interpreting the data, discussing the findings of the study and drafting the manuscript. All authors read and approved the manuscript.

\section{Funding}

This research was funded by Mekelle University, Mekelle, Northern Ethiopia.

\section{Availability of data and materials}

The datasets used for this study could be deposited in publicly available repositories where appropriate and upon reasonable request. All relevant raw data supporting the findings of this study can also be freely available from the corresponding author through email "enemelkamu@gmail.com "or with other means for researchers wishing to use them for non-commercial purposes without breaching participant confidentiality. There will not be any apprehension about the ethical aspect as participant data were de-identified.

\section{Ethics approval and consent to participate}

Ethical approval and clearance were obtained from the institutional review board of Mekelle University, College of Health Sciences. Additional authorization was obtained from Jimma University specialized teaching hospital for data collection. Moreover, verbal informed consent was taken before data collection started. Verbal consent was taken as there is no harm to the study participants because of participating in this study and it was approved by the ethical review board of Mekelle University. To keep the confidentiality of the participants, personal identifiers like the name of the participant was not included in the data collection format. Again, participation was entirely voluntary.

\section{Consent for publication}

Not applicable.

\section{Competing interests}

The authors declare that they have no competing interests.

Received: 19 May 2020 Accepted: 26 October 2020

Published online: 02 December 2020

\section{References}

1. Valentin-Hjorth, J. F., Patou, F., Syhler, N., Vall-Lamora, M. H. D., \& Maier, A. (2018). Design for Health:Towards Collaborative Care. In DS92: Proceedings of the DESIGN 2018 15th International Design Conference (pp. 2775-2786). Design Society. https://doi.org/https://doi.org/10.21278/idc.2018.0506

2. Duty PLS, Williams D. Interprofessional collaboration: a healthcare professional's duty: 2016.

3. What is Collaborative Practice, Alberta health services, 2016.

4. Alisha Karki1, Sara Thapa1 *, BedantakalaThulung1. Attitude towards collaborative care among nurses and physicians at a teaching hospital, chitwan, J Chitwan Med Coll 2018;8(26):47-53. www.jcmc.cmc.edu.np

5. Jenitha S, Verma G. Original article Physician-Nurse collaboration-Is there a discrepancy? 2015:67-77.
6. Smith DC. Midwife - physician collaboration : a conceptual framework for interprofessional collaborative practice. 2015.

7. Elsous A, Radwan M, Mohsen S. Nurses and physicians attitudes toward nurse-physician collaboration : a survey from gaza strip, Palestine. 2017;2017.

8. Franco NP, Cordero MAW. Collaboration Effort between Physicians and Nurses: A Feedback Tool for the Review of the Hospitals. 2017:4(1):19-26.

9. Schmacke N. The Long Way Toward Cooperation : Nurses and Family Physicians in Northern Germany. 2015.

10. Sz H, Ahmad FA, Noh SHM. Nurses' Perceptions and Attitude Toward Nurse-Doctor Relationship at the Tertiary Hospital in Kuala Lumpur. 2018;1(2):1-12.

11. Amsalu E, Boru B, Getahun F, Tulu B. Attitudes of nurses and physicians towards nurse-physician collaboration in northwest Ethiopia : a hospital based cross-sectional study. 2018:1-6

12. Downe S, Finlayson $\mathrm{K}$, Fleming A. Creating a collaborative culture in maternity care. J Midwifery Womens Health. 2010;55(3):250-4. https://doi. org/10.1016/j.jmwh.2010.01.004.

13. Romijn A, Teunissen PW, Bruijne MC De, Wagner C, Groot CJM De. Interprofessional collaboration among care professionals in obstetrical care: are perceptions aligned ? 2018;279-86.

14. Hojat M, Fields S, Veloski JJ, Griffiths M, Cohen MJM, Plumb ID. psychometric properties of an attitude scale measuring physician-nurse collaboration. Eval Health Prof. 1999:22(2):208-20.

15. Zakerimoghadam M, Ghiyasvandian S, Kazemnejad A. Nurse - Physician Collaboration : the Attitudes of Baccalaureate Nursing Students at Tehran University of Medical Sciences. 2015;17(4).

16. Cordero MAW, Alghamdi R, Almojel S, Alhifty E, Khired Z. Physicians and nurses attitude towards physician-nurse collaboration in saudi government hospitals saudi, journal of medical and pharmaceutical sciences. Saudi J Med Pharm Sci. 2018:4(8):871-8.

17. Falana TD, Afolabi OT, Adebayo AM. Collaboration between Doctors and Nurses in a Tertiary Health Facility in South West Nigeria: Implication for Effective Healthcare Delivery. 2016;9(1):165-73.

18. Conn LG, Kenaszchuk C, Dainty K, Zwarenstein M, Reeves S. Nurse - Physician Collaboration in General Internal Medicine: A Synthesis of Survey and Ethnographic Techniques. Health Interprofess Pract. 2014;2(2):1057.

19. Science M, Academy M, Groningen A, Kingdom U. Collaboration of midwives in primary care midwifery practices with other maternity care providers Well Satisfied with Collaboration Less Satisfied with Collaboration. Midwifery. 2017:55:45-52.

20. Sayed KAEL, Sleem WF. Nurse-physician collaboration: a comparative study of the attitudes of nurses and physicians at Mansoura University Hospital. Life Sci J. 2011;8(2):140-6.

21. Elithy ATT, Harmina MK, Elbialy GG. Nurses and Physicians Perceptions of Their Interprofessional Relationships at Alexandria Main University Hospital. J Am Sci. 2011;7:12.

22. Lindeke LL, Sieckert AM. Nurse-Physician Workplace Collaboration. 2015.

\section{Publisher's Note}

Springer Nature remains neutral with regard to jurisdictional claims in published maps and institutional affiliations.

Ready to submit your research? Choose BMC and benefit from

- fast, convenient online submission

- thorough peer review by experienced researchers in your field

- rapid publication on acceptance

- support for research data, including large and complex data types

- gold Open Access which fosters wider collaboration and increased citations

- maximum visibility for your research: over 100M website views per year

At $\mathrm{BMC}$, research is always in progress.

Learn more biomedcentral.com/submissions 